Supplement of The Cryosphere, 10, 1003-1020, 2016

http://www.the-cryosphere.net/10/1003/2016/

doi:10.5194/tc-10-1003-2016-supplement

(C) Author(s) 2016. CC Attribution 3.0 License.

(c) (1)

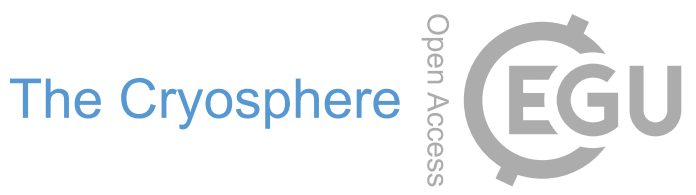

Supplement of

\title{
Past ice-sheet behaviour: retreat scenarios and changing controls in the Ross Sea, Antarctica
}

Anna Ruth W. Halberstadt et al.

Correspondence to: Anna Ruth W. Halberstadt (ar.halberstadt@ rice.edu)

The copyright of individual parts of the supplement might differ from the CC-BY 3.0 licence. 
Supplementary Table 1. Multibeam dataset compilation. NBP1502 cruise data were used here with the permission of J.B. Anderson. The remaining Nathaniel B. Palmer (NBP) cruise data were accessed through the Lamont-Doherty Earth Observatory (marine-geo.org) and the Oden data are available at oden.geo.su.se. Swedish Polar Research = SPR.

\begin{tabular}{|c|c|c|c|c|}
\hline $\begin{array}{l}\text { Cruise } \\
\text { Number }\end{array}$ & Vessel & Multibeam System & Date & $P I$ \\
\hline NBP9801 & NBP & SeaBeam Instruments 2112 & $1 / 16 / 1998-2 / 18 / 1998$ & J.B. Anderson \\
\hline NBP9802 & NBP & SeaBeam Instruments 2112 & 2/22/1998 - 4/2/1998 & S. Honjo \\
\hline NBP9803 & NBP & SeaBeam Instruments 2112 & $5 / 1 / 1998-6 / 17 / 1998$ & M. Jeffries/D. Garrison \\
\hline NBP9807 & NBP & SeaBeam Instruments 2112 & $11 / 1 / 1998-12 / 12 / 1998$ & R. Dunbar \\
\hline NBP9901 & NBP & SeaBeam Instruments 2112 & $12 / 26 / 1998-2 / 4 / 1999$ & M. Jeffries \\
\hline NBP9902 & NBP & SeaBeam Instruments 2112 & $2 / 12 / 1999-3 / 22 / 1999$ & J.B. Anderson \\
\hline NBP9909 & NBP & SeaBeam Instruments 2112 & $12 / 20 / 1999-2 / 9 / 2000$ & J. Bengtson \\
\hline NBP0001 & NBP & SeaBeam Instruments 2112 & $2 / 14 / 2000-3 / 30 / 2000$ & S. Jacobs/T. Kellogg \\
\hline NBP0209 & NBP & Kongsberg EM120 & $12 / 11 / 2002-12 / 30 / 2002$ & S. Cande \\
\hline NBP0301 & NBP & Kongsberg EM120 & $1 / 5 / 2003-1 / 29 / 2003$ & L. Bartek/B. Luyendyk \\
\hline NBP0301A & NBP & Kongsberg EM120 & $2 / 1 / 2003-2 / 18 / 2003$ & P. Bart \\
\hline NBP0301B & NBP & Kongsberg EM120 & $2 / 20 / 2003-2 / 22 / 2003$ & W. Smith/V. Asper \\
\hline NBP0302 & NBP & Kongsberg EM120 & $2 / 24 / 2003-4 / 4 / 2003$ & A. Gordon \\
\hline NBP0305A & NBP & Kongsberg EM120 & $12 / 20 / 2003-12 / 30 / 2003$ & W. Smith \\
\hline NBP0306 & NBP & Kongsberg EM120 & $1 / 4 / 2004-1 / 15 / 2004$ & B. Luyendyk/L. Bartek \\
\hline NBP0401 & NBP & Kongsberg EM120 & $1 / 19 / 2004-2 / 17 / 2004$ & T. Wilson \\
\hline NBP0402 & NBP & Kongsberg EM120 & $2 / 21 / 2004-4 / 6 / 2004$ & M. Visbeck \\
\hline NBP0408 & NBP & Kongsberg EM120 & $10 / 12 / 2004-12 / 6 / 2004$ & S. Jacobs \\
\hline NBP0409 & NBP & Kongsberg EM120 & $12 / 18 / 2004-1 / 21 / 2005$ & R. Kiene/D. Kieber \\
\hline NBP0501 & NBP & Kongsberg EM120 & $1 / 28 / 2005-2 / 13 / 2005$ & A. Gordon \\
\hline NBP0508 & NBP & Kongsberg EM120 & $10 / 26 / 2005-12 / 3 / 2005$ & P. Neale \\
\hline NBP0601 & NBP & Kongsberg EM120 & $12 / 17 / 2005-1 / 24 / 2006$ & G. DiTullio \\
\hline NBP0601A & NBP & Kongsberg EM120 & $1 / 30 / 2006-2 / 2 / 2006$ & W. Smith \\
\hline NBP0602 & NBP & Kongsberg EM120 & $1 / 30 / 2006-2 / 21 / 2006$ & J. Stock \\
\hline NBP0608 & NBP & Kongsberg EM120 & $11 / 3 / 2006-12 / 11 / 2006$ & G. DiTullio \\
\hline NBP0701 & NBP & Kongsberg EM120 & $12 / 22 / 2006-1 / 28 / 2007$ & S. Cande/P. Castillo \\
\hline NBP0702 & NBP & Kongsberg EM120 & $2 / 2 / 2007-3 / 23 / 2007$ & S. Jacobs \\
\hline OSO0708 & Oden & Kongsberg EM122 & $11 / 29 / 2007-1 / 7 / 2008$ & SPR Secretariat \\
\hline NBP0801 & NBP & Kongsberg EM120 & $1 / 9 / 2008-1 / 26 / 2008$ & D. Caron/B. Huber \\
\hline NBP0802 & NBP & Kongsberg EM120 & $1 / 30 / 2008-2 / 20 / 2008$ & D. Caron/P. Bart \\
\hline NBP0803 & NBP & Kongsberg EM120 & $2 / 22 / 2008-3 / 13 / 2008$ & P. Bart \\
\hline NBP1005A & NBP & Kongsberg EM120 & $1 / 13 / 2010-1 / 16 / 2011$ & P. Yager \\
\hline OSO0910 & Oden & Kongsberg EM122 & $2 / 8 / 2010-3 / 12 / 2010$ & M. Jakobsson/J.B. Anderson \\
\hline NBP1005 & NBP & Kongsberg EM120 & $11 / 26 / 2010-1 / 16 / 2011$ & P. Yager \\
\hline OSO1011 & Oden & Kongsberg EM122 & $12 / 8 / 2010-1 / 16 / 2011$ & SPR Secretariat \\
\hline NBP1101 & NBP & Kongsberg EM120 & $1 / 19 / 2011-2 / 15 / 2011$ & J. Kohut/A. Kutska \\
\hline NBP1102 & NBP & Kongsberg EM120 & 2/19/2011 - 4/23/2011 & J. Swift \\
\hline NBP1201 & NBP & Kongsberg EM120 & $12 / 24 / 2011-2 / 11 / 2012$ & D. McGillicuddy \\
\hline NBP1202 & NBP & Kongsberg EM120 & $2 / 11 / 2012-2 / 27 / 2012$ & H. Owen \\
\hline NBP1210 & NBP & Kongsberg EM120 & $1 / 6 / 2013-2 / 9 / 2013$ & K. Halanych \\
\hline NBP1302 & NBP & Kongsberg EM120 & $2 / 12 / 2013-4 / 5 / 2013$ & D. Hansell/X. Yuan/G. Kooyman \\
\hline NBP1310B & NBP & Kongsberg EM120 & $12 / 3 / 2013-1 / 23 / 2014$ & K. Arrigo/R. Aronson \\
\hline NBP1502A & NBP & Kongsberg EM122 & $1 / 23 / 2015-3 / 20 / 2015$ & J.B. Anderson \\
\hline
\end{tabular}

